PERSONALPLANUNG

\title{
Kosten minimieren, Ressourcen optimieren
}

\author{
Dieter Dienwiebel
}

Die TÜV Akademie GmbH hat ein Assessment Center entwickelt, das individuelle Aussagen über Fachkompetenzen, Methodenkompetenzen, soziale Kompetenzen, personale Kompetenzen, Kulturtechniken und psychomotorische Merkmale der Teilnehmer gestattet.

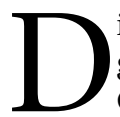
ie TÜV Akademie GmbH gehört zur TÜV Rheinland Group und unterliegt nicht weniger als andere Gesellschaften und Träger dem Wettbewerbsdruck. Sie ist traditionell in der Berufsausbildung, der Fort- und Weiterbildung sowie der Berufs- und Benachteiligtenförderung tätig.

Dabei kommen vereinzelt eignungsdiagnostische Instrumente wie Profiling und Assessment Center zum Einsatz. Um langfristig die Entwicklungen im Bildungs- und Ausbildungsmarkt mit zu bestimmen und die Nase vorn zu haben, sollten die Erfahrungen und Kräfte gebündelt und von Grund auf eine eigenständige Entwicklung der TÜV Akademie GmbH den Niederlassungen und Mitarbeitern für die Assessment Center-Technologie zur Verfügung gestellt werden.

Die Entscheidung fiel auf ein modularisierbares Assessment Center, um es möglichst vielseitig adaptieren zu können und den Mitarbeitern in den Projekten fachlich abgesicherte Handlungsoptionen zur Verfügung zu stellen. Es ist also kein einzelnes Assessment Center vorgesehen, sondern es ist ein System von Assessment Centern in Angriff genommen worden. Dabei sind die langjährigen positiven Erfahrungen der Mitarbeiter bei der Anwendung einzelner eignungsdiagnostischer Instrumente eine nicht unerhebliche Voraussetzung für den Erfolg.
Nunmehr ist eines der ersten Assessment Center in diesem System für die Niederlassungen des Unternehmens entwickelt worden. Es gestattet, individuelle Aussagen über Fachkompetenzen, Methodenkompetenzen, soziale Kompetenzen, personale Kompetenzen, Kulturtechniken und psychomotorische Merkmale zu treffen. Hierzu werden unterschiedliche Instrumente einschließlich Persönlichkeitstests eingesetzt.

Die Kompetenzen und Kulturtechniken sind in 44 Dimensionen strukturiert, die aus minimal 130 und optimal 300 Einzelangaben für jeden Teilnehmer im Assessment Center hergeleitet und in Endgutachten zusammen mit Interviews und Feedbacks verdichtet und verarbeitet werden. Das Verfahren erfüllt folgende Parameter:

- Es ist ein Assessment Center, das als eignungsdiagnostisches Instrument standardisiert und normiert ist. Es erfüllt die anspruchsvolle Norm DIN 33430. Gleichzeitig werden die Standards des Arbeitskreises Assessment Center e. V. berücksichtigt.

- Das Instrument ist komplex und umfassend entwickelt. Im Moment liegt das Assessment Center zum Einsatz in der Benachteiligten- und Berufsförderung vor. Es ist von vornherein für die Adaption durch den Einsatz mit weiteren Modulen ausgelegt. Im Einzelnen heißt das, die Spannbreite des Einsatzes kann sein:

- derzeitig von der Benachteiligtenund Berufsförderung und bis hin zum Einsatz für die Berufsausbildung, dem zweiten Bildungsweg oder auch für Privatschulen und

- zukünftig zur Personalauswahl und Personalentwicklung in der Privatwirtschaft, insbesondere in mittelständigen Unternehmen.

- Die Datenerfassung und Datenauswertung erfolgt mit einer eigens

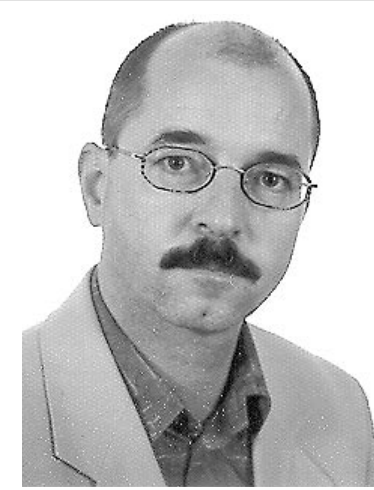

Dieter Dienwiebel ist DiplomSozialpädagoge und Magister des Sozialmanagements. Er ist freiberuflich bei Bildungsträgern beschäftigt, hat Lehraufträge an Hochschulen übernommen und ist in Privatunternehmen als Trainer tätig. In Zusammenarbeit mit Diplombetriebswirten und Softwareingenieuren entwickelt er Assessment Center für freie Träger.

E-Mail dieter.dienwiebel@prowork.cc

entwickelten Software, die gleichzeitig zur Unterstützung der Gutachtenerstellung verwendet wird.

- Die Leistungsparameter bei der Anwendung der Assessment Center in den einzelnen Niederlassungen des Unternehmens, die Ergebnisse und auch die Qualitätskennziffern können zeitnah bestimmt und in der Unternehmenspolitik eingesetzt werden.

- Die Entwicklung der Assessment Center wird als Projektmanagements nach DIN 69901 vorangetrieben. Es wird die Projektentwicklung systemisch mit einer breiten Mitarbeiterausbildung verbunden. Das Ergebnis ist ein beachtlich hoher Identifikationsfaktor, besonders bezüglich der Corporate Identity.

Die Assessment Center-Technologie fordert professionelle Standards und Personalqualifizierung. Das ergibt sich zunächst aus dem ethischen Anspruch, Aussagen über Teilnehmer und Beteiligte in hoher Güte zu entwickelt und verantwortungsvoll mit den Betroffenen zusammenzuarbeiten.

Aber weiterhin ergibt sich diese Forderung aus den betriebswirtschaftlichen Belangen eines Unterneh- 
mens. Der Leistungserstellungsprozess, der für die Eignungsdiagnostik erforderlich ist, erzeugt beträchtliche Aufwendungen. In der freien Wirtschaft und in vielen höheren Bildungseinrichtungen ist es unumstritten, dass sich diese Aufwendungen lohnen.

Ein Assessment Center muss mit dem Unternehmen systemisch verbunden werden. Es bedarf ausgereifter Installationsstrategin, des Spezialisteneinsatzes und der Förderung engagierter Mitarbeiter, die Gestaltungsbedürfnisse und Selbstanspruch miteinander verbinden. Die Verknüpfung der Eignungsdiagnostik mit Bildungs- und Ausbildungsprojekten bedarf eines umfangreichen organisatorischen und fachlichen Aufwandes. Eine besondere Herausforderung ist, die Güte der Assessment Center zu bestimmen.

Projekte, die von den Arbeitsagenturen oder regionalen »Einkaufszentren« im Zusammenhang mit der Assessment Center-Technologie ausgeschrieben werden, sind nicht mehr wie bisherige Projekte der Bildung, Umschulung oder Qualifizierung zu installieren und durchzuführen. Dieser Umstand wurde für Träger im Marktsegment der Berufsund Benachteiligtenförderung mitunter schmerzhaft und immer deutlicher wahrgenommen. Der bei vielen Trägern verbreitete Mythos, bisher haben wir alles geschafft, also schaffen wir das auch, scheitert hier. Es ist ein neuer Technologieanspruch notwendig.

Diese Dimensionen werden in der TÜV Akademie $\mathrm{GmbH}$ berücksichtigt. Um die Kosten zu minimieren und gleichzeitig die Ressourcen zu optimieren wird ein Coaching realisiert. In Zusammenarbeit mit PROWORK Consultingbüro für Sozialmanagement und Personalentwicklung wurden so folgende Parameter miteinander verbunden:

- die Fachberatung in der TÜV Akademie GmbH

- die Beratung und Schulung von Mitarbeitern in den Ausbildungszentren und Niederlassungen

- die Schulung und Qualifizierung der Projektleiter, der Beobachter, von Interviewern und die Schulung zur Gutachtenerstellung

- die Entwicklung und Bereitstellung von Software zur Datenerfassung und Datenverarbeitung sowie zur Verschriftlichung von Gutachten in den Assessment Center

Diese Parameter betreffen also das Projektmanagement, die Mitarbeiterqualifizierung und Mitarbeiterpartizipation, die konsequente Einbindung des mittleren Managements und die Ausstattung mit effizienter und den spezifischen Ansprüchen genügenden Software.

Die Erfahrungen besagen, dass bei der Durchführung der Assessment entwickelt wird. Im Einzelnen und explizit werden sie auch in DIN 33430 angeführt und kommentiert. Es ist im Interesse der Teilnehmer, der Mitarbeiter, der externen Auftraggeber und des Unternehmens, die Validität und die Reliabilität zu bestimmen.

Es ist von Interesse, die Objektivität des Instrumenteneinsatzes zu sichern. Diese Entwicklung ist für das angestrebte Sozialcontrolling von beachtlichem Wert. Es ist Ziel, die

\section{"Die Entwicklung von Assessment Centern bei freien Trägern fordert das ganze Sozialunternebmen"}

Center und deren Auswertung die Mitarbeiter hoch engagiert sind. Hier entsteht gewissermaßen eine Eigendynamik. Die Mitarbeiter legen vor allem in den Personalkompetenzen zu. Das Lernvolumen für Mitarbeiter ist kaum zu unterschätzen. Zugleich können Probleme der Lehr- und Ausbildungsinhalte als auch der psychosozialen Begleitung oder auch des Case Managements für die Projektteilnehmer verbessert werden. Diese Effekte werden vom Management der freien Träger natürlich gern entgegen genommen und willkommen geheißen.

Die Assessment Center sind aber nicht nur leistungsintensiv, sie sind auch anerkanntermaßen eine der hochwertigsten und anspruchsvollsten eignungsdiagnostischen Instrumente. Die Gütebestimmung gehört dazu. Sie sollte von freien Trägern in Zukunft verstärkt abgefordert werden. Diese Herangehensweise fordert im Projektmanagement die Spezialistenarbeit, ein komplexes Herangehen an die Eignungsdiagnostik, die Berücksichtigung spezifischer Bedingungen bei freien Trägern in der Sozialwirtschaft und natürlich eines ausreichenden Durchhaltevermögens und Engagements von Mitarbeitern und Führungskräften.

Von Anfang an besteht in der TÜV Akademie GmbH die Absicht, die Technologie im Unternehmen so zu installieren, dass die Assessment Center-Technologie nach den anerkannten Kriterien zur Gütebestimmung
Wirksamkeit der Technologie klar zu bestimmen, Entscheidungshilfen für die Entwicklungsrichtungen der Assessment Center und für die einzelnen Ausbildungs- und Bildungssegmente der TÜV Akademie GmbH bereitzustellen.

\section{Resümee}

Die Herausforderungen eines komplexen Systems von Assessment Center bei einem freien Träger in der Sozialwirtschaft schaffen Übergänge zur Organisationsentwicklung und Personalentwicklung. Die Entwicklung will ausgewogen und differenziert vorangetrieben werden. Die TÜV Akademie GmbH hat die Entwicklung aufgenommen und hat mit Partnern und Spezialisten bereits wichtige Schritte getan. Sie setzt sich hier für die Interessen der Betroffenen, ihrer Mitarbeiter und für die Ausgestaltung einer wettbewerbsorientierten sozialen Infrastruktur ein. 